\title{
Fluorochrome Dye
}

National Cancer Institute

\section{Source}

National Cancer Institute. Fluorochrome Dye. NCI Thesaurus. Code C503.

A class of dyes when excited by light of certain wavelengths, emit photons and become fluorescent. Fluorescence gives fluorochromes imaging characteristics and are used to label or stain biological structures. 